\title{
REVIEW
}

\section{Abstinence Design}

\section{Qiang $\mathbf{W u} \mathbf{u}^{*}$}

Wuyi University, Wuyishan, Fujian, 354300, China

\begin{tabular}{|c|c|}
\hline ARTICLE INFO & ABSTRACT \\
\hline Article history & \multirow{10}{*}{$\begin{array}{l}\text { Abstinence design is a design that helps people to get rid of bad habits } \\
\text { or hobbies for self-management purposes, which seems to limit people's } \\
\text { "freedom" and is contrary to the idea of designing "people-oriented". } \\
\text { However, it is not difficult to find out that only the design that can resist } \\
\text { "human desire" is the design of "true freedom." Abstinence design has } \\
\text { the idea of green design and minimalist design. It is more in line with } \\
\text { the Chinese culture and philosophy, and has positive significance for the } \\
\text { improvement of the quality of individuals and even the whole nation. } \\
\text { In designing abstinence products, in addition to the degree of desire and } \\
\text { need to be treated differently, more guidance on design methods, such as } \\
\text { fun, gamification, peak-end principle, etc., is required to enable users to } \\
\text { achieve self-management more effectively. }\end{array}$} \\
\hline Received: 18 October 2019 & \\
\hline & \\
\hline Revised: 25 October 2019 & \\
\hline Accepted: 9 April 2020 & \\
\hline Published Online: 16 April 2020 & \\
\hline Keywords: & \\
\hline Abstinence design & \\
\hline Freedom & \\
\hline Design method & \\
\hline
\end{tabular}

\section{The Definition of Abstinence Design}

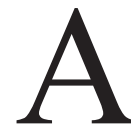
bstinence design, as the name implies, it is based on the design concept of "abstain" and "restrain". "Abstain" is the meaning of quit, thus quit bad habits and hobbies. "Restrain" means self-discipline and self-restraint. Therefore, abstinence design is a design that helps people to quit bad hobbies and guide people to self-discipline and confront the "desire" with it.

\section{Freedom and Abstinence}

Freedom and abstinence are two seemingly contradictory nouns. Humans pursue freedom and exclusion is constrained. Abstinence is also a constraint but this is not the case. Humanism is advocated in Western countries, and in humanism, "liberalism" is the mainstream thinking. Liberalists believe that everyone's inner experience is important and can make the world rich and colorful, so everyone has the right to freely express. Liberalists believe that people have an inseparable "self", this true self is completely free, the so-called "free will." Moreover, no one knows himself better than himself, so no one needs to make a decision for me. ${ }^{[1]}$

But through a series of scientific experiments, this liberal view is wrong. In particular, scientists have shown that the free will of human beings is actually the result of the control of human consciousness by desires, and that human desires are hardly controlled by consciousness. As Yuval Noah Harari said in "Homo Deus: A Brief History of Tomorrow": "Human consciousness is a collection of thoughts, feelings, and desires. Every moment, every desire rises and disappears in our brains. Your decision may be the result of a variety of different desire negotiations, but what are the desires to attend this negotiation, you

\footnotetext{
*Corresponding Author:

Qiang $W u$,

Wuyi University, No. 358 Baihua Road, Wuyishan, Fujian, 354300, China,

E-mail: 592088156@qq.com

Fund Project:

Project No. XD201811S on Green Design and Application of Fiber-Based Material Waste of Wuyi Rock Tea.
} 
can't control it at all. The so-called liberalism seems to be synonymous with "desireism."

It seems that we have seen the struggle of human nature. Some thoughts and religions have proposed rules and doctrines. These are abstinences that help people regulate their behavior and avoid the harm caused by desire. Behavior within abstinence needs to be constrained, and behavior outside of abstinence is true freedom. Therefore, in the religious teachings of Buddhism, true freedom can only be obtained through abstinence. Historical philosophers also believe that the subject gains freedom by escaping the external constraints of the object. From this point of view, freedom and abstinence are not just gram, but also born.

\section{Why Abstinence Design Is Needed?}

Modern design emphasizes "humanized design" or "people-oriented" design. That is, in the design process, "the user's needs, capabilities, and behaviors are analyzed first, and then the design is used to meet people's needs, abilities, and behaviors." This design concept is incomprehensible. The design needs to serve people. The people are the main body of design, but the problem is exactly in people. Human nature itself has shortcomings. In the case of greed, human desires are everywhere, ranging from sleep control (sleepiness, bed-riding) to the infinite capture of the earth (excessive use of resources), which is not a manifestation of human desire. If allowing unrestrained indulgence of desire, there will be a huge damage to both individuals and humans. If following this "humanized" design then the consequences will only be worse.

In fact, human beings themselves have realized the negative nature of desire, but because bad behavior habits and desires are difficult to quit and control, and will give birth to many "excuses" to "justify" their habits and desires, leading to the failure of self-control action. Therefore, external factors are needed to help human abstinence to counter the desire to achieve management of human beings, to meet the design of people to quit bad habits and self-management, that is, abstinence design needs to emerge.

\section{What Is the Significance of Abstinence De- sign?}

\subsection{Abstinence Design Is a Green Eco-design in a Broad Sense}

Abstinence design emphasizes not to "indulge" to quit desire and self-control. The destruction of ecology, from the perspective of human nature, is the endless demand and waste of human beings to nature, and the destruction of the environment cannot be self-monitored. For example, people's use of tap water, although the slogan of promoting water conservation in life is already familiar, but in real life, for various reasons, domestic water is still demanding. Therefore, the design to help and guide people to save water is undoubtedly an abstinence design that helps people self-control and waste water. Therefore, the design of abstinence belongs to the category of green design in some aspects.

\subsection{Abstinence Design Belongs to a Minimalist Design Idea}

People sometimes buy a lot of unwanted things because they want to follow the trend or buy cheaply or to satisfy their shopping desires. For example, a woman always feels that there is a set of clothes that can be worn in the closet, even though the wardrobe is full. Although the home has become a showroom for shoes, there is no shoe in the shoe that can match clothes. Then the design to help women's rational consumption is undoubtedly an abstinence design. This kind of design will reach people's minimalist life concept to some extent, which makes people truly recognize their needs, cherishes what they really need in life, reduces the number of items around them and improves quality and taste.

\subsection{Abstinence Design Is More in Line with Chi- nese Philosophy and Cultural Thoughts}

Western ideas are mainly humanistic, that is, people-oriented, emphasizing free will.

This kind of will is difficult to define. Once it is detached from the scope, it will lead to the growth of self-interested ideas and the infinite expansion of desires in human nature. In "The Book of Rites Record of Music", there are words: "The human beings are also those who destroy the heavens and the poor." Then there is a rebellious scam, and there is obscenity and chaos. The socalled "abolishing the heavenly principles and reaching the greatest desires of the people" here means annihilating the heaven principles and doing whatever they want.

The opposite of China's philosophical thinking is to emphasize "heaven" and the status of the person is at the second place. In the traditional Chinese thoughts, there are Confucian ideas including "the theory of heaven and man"; and "self-cultivating"; Buddhism doctrines freed from human nature with "desire", "anger", and "ignorance"; and the essence of Taoism that "knowing contentment and stop". These abstinence ideas are rooted in the soil of Chinese thought and are accepted by the Chinese. 
The abstinence design corresponding to this is more in line with China's ideological and moral standards and is the design of more "Chinese thoughts". ${ }^{[5]}$

On the other hand, China's ideological and cultural structure has a "tradition" in which parents take care of their children. Thoughts such as "the life of the parents" have made many parents in China control the behavior of their children. Excluding the child's rebellious attitude towards this behavior, just look at the consequences of this behavior, which has made many of the younger generation in China out of the bondage of their parents and become self-sufficient and self-disciplined. Therefore, there will be many phenomena of accompanying parents chasing foreign countries to supervise and accompany their children. There are also many cases in which Chinese students leave their parents and are unemployed abroad and ruined their studies. Excluding the reasons for the dependence of parents' excessive interference, self-restraint is insufficient, and the lack of self-control of desire is also an important factor. ${ }^{[4]}$ Therefore, abstinence design has a good healing effect in helping the younger generation with poor self-control, and it is more in line with Chinese culture and national conditions.

\subsection{Abstinence Design Helps Individuals Com- plete Self-management to Improve the Quality of Individuals and Even the Entire Nation}

Self-management is an important topic after the 90s. The topic of "bubble beer", which has been discussed hotly before, and the growing number of "health institutions" and "self-management" institutions all show the importance of self-management. These are also important contents of abstinence design. It not only improves people's physical fitness, but also plays an important role in the reshaping and improvement of spirit and personality, and has an intersection with healthy design. (Such as physiological management: the maintenance of the body, reminding to strengthen the movement; psychological management: such as anger, decompression, etc.). In addition, abstinence design also has management of time and life planning. In the long run, abstinence design also allows the audience to "self-cultivating" while at the same time, more "family-regulating", "state-ordering", and "world-governing" to achieve higher goals and realms of life.

\section{The Abstinence of the Strength of "Desire" and the Willingness Level to Self-restraint}

According to the intensity of the desire and self-abstinence, from weak to strong, it can be roughly divided into three levels. Level 1: Guided-by as the main. Advocating correct concepts, public opinion and trend orientation, attracting attention and raising awareness. For example, reducing waste and raising environmental awareness. Level 2: Self-discipline as the main. Repeated restraint becomes a habit, emphasizing the self-restraint force of cultivating people. For example, in order to lose weight, restrain excessive intake of sugar, adhere to fitness and so on. Level 3: Abstaining as the main. Rely on external power to get rid of addicted bad habits, such as Internet addiction, addiction and so on. Therefore, in the design of abstinence, it is necessary to distinguish the design according to different levels and strengths.

\section{What Are the Methods of Abstinence De- sign?}

\subsection{Visually Quantify or Image Transform the De- sign}

Data and quantification allow users to feel guilty in "indulgence" and a sense of accomplishment in "abstinence". Such as the WeChat sports application in social software WeChat, The user's movement is directly quantified as "steps", and the number of steps of the friends in the software is ranked, which has increased the "sense of achievement" of the sports user and the "pressure feeling" of the user with less exercise. Similar software Baidu Map also has statistics on the number of steps. The energy consumed in the search for a location in the map translates directly into a food image, allowing the user to feel their movements more intuitively while walking, rather than simply boring kilometers in fitness equipment. Moreover, such image transformation can also become a more intuitive basis for users to consider the diet. Visual data quantification and image transformation are one of the commonly used techniques of abstinence design, which will enable consumers to better understand the results of "abstinence" and increase the motivation to quit negative desires.

\subsection{Accumulate "Micro Habits" with "Single Steps"}

Pay attention to the long-term change of abstinence, not the short-term effect of "explosive power". "Micro-habitation" is a small positive behavior that is small enough to fail small goals. For example, if you want to get a good figure, do at least one push-up every day. It is because of the small goal that it will not put pressure on people. Moreover, it can help people overcome the two major obstacles of habit cultivation: (1) unable to start action, lack of courage and motivation; (2) unable to persist and lack 
willpower. Therefore, through abstinence design, let users make a good "micro habit" through a little change every day, forming a "inertia" of life, thus overcoming inertia. In the subtle, the goal of self-discipline and self-management is achieved by a planned "single steps" accumulation.

\subsection{Use Peak Value-Ending Rules in Design}

In Yuval Noah Harari's "Homo Deus: A Brief History of Tomorrow", there is a left and right brain experiment. The experiment proves that "narrative self" will "deceive" and "experience self". The "narrative self" is the most memorable part of the experience's climax and ending when assessing an experience and the rest of the experience is chosen to be ignored. This proves that "experiencing self" is easily deceived by "narrative self" and can only remember the climax and the end. For example, during surgery, by giving the patient a good experience at the end of the surgery, this will give the patient a better feeling and feedback on the surgery. Therefore, the peak value-ending rules are applied to the abstinence design. If the design and use experience is nearing the end, add emotional design elements or reduce the intensity of abstinence, and reduce negative emotions such as conflicts and boredoms of users.

\subsection{Let the Abstained Things Produce the Neces- sary Utility or Produce a Stronger Temptation}

Some products are designed to produce additional features. For example, the trunk itself has the function of loading luggage, but it has the function of a seat when the user is exhausted. At the same time, the reason why things are tempting to people cannot be quit because humans have demand for them. According to Maslow's hierarchy of needs, demand can be divided into five categories: physiological needs, security needs, love and belonging, respect and self-realization. If you give a product a new feature to meet a lower level, it will reduce the temptation of the original function of the thing. For example, a "good dining table" designed by IKEA must allow the diners to place the mobile phone under the hot pot table, and the stove can generate heat to help the diners to quit the mobile phone. When the physiological needs (hunger) need to be met, the demand for mobile phones (the need for security needs, love and belonging) is well removed. ${ }^{[3]}$

\subsection{Design the Process of "Abstinence" to Make It Fun and Gamified}

The process of abstinence was boring and even painful at first, resulting in negative emotions such as resistance and depression, which made the "abstinence" plan fail. Fun design and gamification design can alleviate and improve many negative emotions and convey humorous, light and happy information. According to the peakend rule, the fun and gamified design will give the user a positive feeling of novelty and pleasure in the later stages of use, which allows the user to receive "fun" and "fun" signals at the end of the abstinence process, so that they can experience happiness in the abstinence process and gain joy after the success of abstinence. Such as a running alarm clock, this English name "hide and seek" alarm clock known as the invincible opponent of lie-in. At the time when the alarm clock was set up, it was not only the ringing of the alarm, but also the running of the wheels on the alarm clock and "hiding", which makes the user have to get up immediately and get the most out of it to turn off the alarm clock, and the process of catching is like playing a "hide and seek" game. However, the user gradually wakes up during the chase process, and even enjoys the game during the later stages of product use. In the long run, naturally, in addition to the habit of sleeping late, the body clock that wakes up early and gets up early.

\subsection{Auxiliary Abstaining by Changing Behavioral Inertia by Using Alternatives in Behavioral Hab- its}

If the behavior exists and continues in inertia, it will eventually become a habit. Behavioral inertia is usually divided into good behavioral inertia (such as washing hands before and after meals, going to bed early and getting up early) and bad behavior habits (smoking, alcoholism, gambling, etc.). This inertia is manifested in behavioral activity, and if not intentionally changed, this behavior will last for a long time. At the same time, habits have a strong inertia on the way of behavior. For example, when a person controls smoking, even if he knows that he does not carry tobacco, people will unconsciously experience inertia such as smoke, fingers and cigarettes. In order to quit this behavior, the behavioral action, or the manifestation of the behavior, can be replaced by the behavioral substance to achieve the purpose of quit. If you quit smoking, the first step can be to try to replace smoking by chewing gum, eating melon seeds, drinking tea, and so on. The second step can maintain the "smoking" behavior, but replace the tobacco with e-cigarette or a harmless but disgusting spice as a burning substance to reduce the attractiveness of the "smoking" behavior, or to produce a strong and sustained aversion, and ultimately achieve the purpose of smoking cessation. 


\subsection{Use Computer Algorithms to Conduct Absti- nence Design}

\section{The Future Development Trend of Absti- nence Design}

Abstinence design is a branch of modern design development. Although the design community has not defined the design concepts of abstinence design such as interaction design, green design and emotional design. However, as people's material life has developed at a higher and faster rate, people need to control and abandon their bad habits caused by high material life. ${ }^{[2]}$ Because greed is one of humanity's human nature, the "humanized" design that satisfies people's needs will gradually narrow into the dead end in the near future. At that time, people had to consider what is true freedom and "humanization". Perhaps, only through abstinence can we find true freedom to meet the "humanized" design that humans really need.

\section{References}

[1] Jiwei Ci. Difficulties and Truths in Literature[J]. Literary Review, 1989(02). (in Chinese)

[2] Jiwei Qin. Research on Contemporary College Students' Self-Discipline Awareness Education[D]. Hunan Normal University, 2014. (in Chinese)

[3] Yongxiang Ma. Management Is the Two-way Construction of the Law And Self-discipline[J]. Jiangsu Education, 2017(04). (in Chinese)

[4] Feng Lu, Wei Xiao, ed. Introduction to Applied Ethics[M]. Contemporary China Publishing House,2002. (in Chinese)

[5] Ning Han, Anguo Xue, Fangming Jiao, Jiaojiao Lu. Research on the Cultivation of Young Citizens' Quality Based on the Traditional Culture Discarding Perspective[J]. Yangtze River Series, 2017(01). (in Chinese) 